\title{
NONLINEAR ELLIPTIC EQUATION WITH VARIABLE EXPONENTS AND MEASURE OR $L^{m}$ DATA
}

\section{F. MOKHTARI ${ }^{1}$, K. BACHOUCHE ${ }^{2}$ and H. ABDELAZIZ ${ }^{3}$}

${ }^{1}$ Laboratoire des EDP

ENS-Kouba, Alger

Department of Mathematics and Informatics

University of Benyoucef Benkhedda (Alger 1)

Alger

Algeria

e-mail: fares_mokhtari@yahoo.fr

${ }^{2}$ Laboratoire TPFA

ENS-Kouba, Alger

Department of Mathematics and Informatics

University of Benyoucef Benkhedda (Alger 1)

Alger

Algeria

e-mail: kbachouche@gmail.com

${ }^{3}$ Laboratoire des EDP

ENS-Kouba, Alger

Faculty of Mathematics and Informatics

University of M'sila

Algeria

e-mail: hellal.ens@gmail.com

2010 Mathematics Subject Classification: 35J67, 35D30, 35J05.

Keywords and phrases: elliptic equations, weak solution, variable exponent, measure or $L^{m}$ data.

Received October 8, 2015

(C) 2015 Scientific Advances Publishers 


\begin{abstract}
In this work, we prove existence and regularity of weak solutions for a class of nonlinear elliptic equations with variable exponents and measure or $L^{m}$ data, with $m$ being small. The functional setting involves Lebesgue-Sobolev spaces with variable exponents.
\end{abstract}

\title{
1. Introduction
}

This work is devoted to the study of the nonlinear elliptic problem

$$
\begin{cases}-\operatorname{div}(\widehat{a}(x, u, D u))=\mu, & \text { in } \Omega, \\ u=0, & \text { on } \partial \Omega,\end{cases}
$$

where $\Omega$ is a bounded open subset of $\mathbb{R}^{N}(N \geq 2)$ with Lipschitz boundary $\partial \Omega$, the right-hand side $\mu \in M(\Omega)$ is a Radon's bounded measure on $\Omega$ or in $L^{m}$, and $\hat{a}: \Omega \times \mathbb{R} \times \mathbb{R}^{N} \rightarrow \mathbb{R}^{N}$ is a Carathéodory function and satisfying, a.e. $x \in \Omega$ and $\forall u \in \mathbb{R}$ and $\forall \xi, \xi^{\prime} \in \mathbb{R}^{N}$, the following:

$$
\begin{gathered}
\widehat{a}(x, u, \xi) \xi \geq \alpha|\xi|^{p(.)}, \quad \widehat{a}(x, u, \xi)=\left(a_{1}, \ldots, a_{N}\right) ; \\
|\widehat{a}(x, u, \xi)| \leq \beta\left(h+|u|^{p(.)-1}+|\xi|^{p(.)-1}\right) ; \\
\left(\widehat{a}(x, u, \xi)-\widehat{a}\left(x, u, \xi^{\prime}\right)\right)\left(\xi-\xi^{\prime}\right)>0, \quad \xi \neq \xi^{\prime},
\end{gathered}
$$

where $\alpha, \beta$ are strictly positive real numbers, $h$ is a given positive function in $L^{p^{\prime}(.)}(\Omega)$, and the variable exponent $p():. \bar{\Omega} \rightarrow(1,+\infty)$ is continuous function such that

$$
2-\frac{1}{N}<p(x)<N, \quad \text { for all } x \in \bar{\Omega} \text {. }
$$

As a prototype example, we consider the model problem

$$
\begin{cases}-\operatorname{div}\left(|D u|^{p(x)-2} D u\right)=\delta, & \text { in } B, \\ u=0, & \text { on } \partial B,\end{cases}
$$


where $\delta$ is the Dirac measure at the origin, $p($.$) as in (1.4), and$

$$
B=\left\{x \in \mathbb{R}^{N}|| x \mid<1\right\} .
$$

Variable exponents involve today in various branches of applied science. In some cases, they provide realistic models for the study of natural phenomena in electro-rheological fluids and an important applications are related to image processing. We refer the reader to ([6], [13], [14], [15]) and the references therein. Clearly, the nonlinearity of (1.5) is more complicated than nonlinearity of the $p$-Laplacian. As the exponent which appear in (1.5) depends on the variable $x$, the functional setting involves Lebesgue and Sobolev spaces with variable exponent $L^{p(.)}(\Omega)$ and $W_{0}^{1, p(.)}(\Omega)$. In particular, if $\widehat{a}(x, u, \xi)=\widehat{a}(\xi)$, with $\widehat{a}(\xi)$ the vector field whose components are $|\xi|^{p(.)-2} \xi$ and $p($.$) is a continuous$ function on $\bar{\Omega}$ such that $p()>.2-\frac{1}{N}$, the existence of renormalized solution for an elliptic problem with $L^{1}$ data on $\Omega$, was proved in [2]. Under the additional hypothesis that the exponent variable $p()>$.1 is log-Hölder continuous, that is,

$\exists M>0:|p(x)-p(y)| \leq-\frac{M}{\ln (|x-y|)} \forall x \neq y \in \Omega$ such that $|x-y| \leq 1 / 2$,

the existence and uniqueness of both entropy solutions and renormalized solutions for the $p(x)$-Laplacian equation with a signed measure in $L^{1}(\Omega)+W^{-1, p^{\prime}(.)}(\Omega)$, is proved in [17]. Remark here that the Dirac mass is note in $L^{1}(\Omega)+W^{-1, p^{\prime}(.)}(\Omega)$, and there is a counterexample in [5] showing why the definition of the entropy solution is note suitable for uniqueness if the right-hand side $\mu=\delta$. If the variable exponent $p($.) satisfies the log-Hölder continuity condition (1.6), $\mu \in L^{1}(\Omega)$, and the function $\widehat{a}$ is independent of $u$, similar results for the notion of entropy 
solution are established in ([16], [18]). When the boundary value condition is a Neumann boundary condition in the context of variable exponent and $\mu \in L^{1}(\Omega)$, the existence and uniqueness of weak and entropy solution of class of elliptic equations has been proved in [3]. An existence result of a renormalized solution for the $p(x)$-Laplacian equation with Neumann nonhomogeneous boundary conditions and $L^{1}$ data is established in [1]. In the constant case $p()=.p>2-\frac{1}{N}$, the existence of a distributional solution $u$ of $(\mathrm{P})$ in the space $W_{0}^{1, q}(\Omega)$ for all $q \in\left[1, \frac{N(p-1)}{N-1}\right)$ has been proved in [4]. If the variable exponent $p($.$) is$ assumed to be merely continuous function, the techniques used in [16] (or in [17]) do not work. Therefore, the study of problem (P) is a new and interesting topic. Inspired by [2], we prove the existence of weak solution for the problem $(\mathrm{P})$ with right-hand side is measure, and the variable exponent $p($.$) as in (1.4). We prove also, the existence result for the$ problem (P) when the datum $\mu$ is assumed to be in $L^{m}(\Omega)$ with $p: \bar{\Omega} \rightarrow$ $(1, \infty)$ is a continuous function and

$$
1+\frac{1}{m}-\frac{1}{N}<p(x)<N, \quad \text { for all } x \in \bar{\Omega},
$$

where

$$
1<m<\frac{N p(x)}{N p(x)-N+p(x)}, \quad \text { for all } x \in \bar{\Omega}
$$

The main steps of the proof consist of obtaining uniform estimates for suitable approximate problems and then passing to the limit. There are essentially two difficulties introduced in treating nonlinear elliptic operators $A u=-\operatorname{div}(\widehat{a}(x, u, D u))$ instead of the Laplacian with variable exponents. The first one is to obtain uniform estimates on the solution $u$ and the gradients $D u$. The second difficulty is to pass to the limit when the nonlinearity of $A$ depends on $u$ and $D u$. 


\section{Mathematical Preliminaries}

In this section, we first recall some facts on variable exponent spaces $L^{p(.)}(\Omega)$. We refer to ([7], [9]) and the references therein, for further properties of variable exponent Lebesgue-Sobolev spaces.

Let $p: \bar{\Omega} \rightarrow[1, \infty)$ be a continuous function. We denote by $L^{p(.)}(\Omega)$ the space of measurable function $f(x)$ on $\Omega$ such that

$$
\rho_{p(.)}(f)=\int_{\Omega}|f(x)|^{p(x)} d x<+\infty .
$$

The space $L^{p(.)}(\Omega)$ equipped with the norm

$$
\|f\|_{p(.)}:=\|f\|_{L^{p(.)}(\Omega)}=\inf \left\{\lambda>0 \mid \rho_{p(.)}(f / \lambda) \leq 1\right\}
$$

becomes a Banach space. Moreover, if $p^{-}=\inf _{x \in \bar{\Omega}} p(x)>1$, then $L^{p(.)}(\Omega)$ is reflexive and the dual of $L^{p(.)}(\Omega)$ can be identified with $L^{p^{\prime}(.)}(\Omega)$, where $\frac{1}{p(x)}+\frac{1}{p^{\prime}(x)}=1$. For any $u \in L^{p(.)}(\Omega)$ and $v \in L^{p^{\prime}(.)}(\Omega)$, the Hölder type inequality

$$
\left|\int_{\Omega} u v d x\right| \leq\left(\frac{1}{p^{-}}+\frac{1}{p^{\prime}}\right)\|u\|_{p(.)}\|v\|_{p^{\prime}(.)} \leq 2\|u\|_{p(.)}\|v\|_{p^{\prime}(.)},
$$

holds true.

We define also the Banach space $W_{0}^{1, p(.)}(\Omega)$ by

$$
W_{0}^{1, p(.)}(\Omega)=\left\{f \in L^{p(.)}(\Omega),|D f| \in L^{p(.)}(\Omega) \text { and } f=0 \text { on } \partial \Omega\right\},
$$

endowed with the norm $\|f\|_{W_{0}^{1, p(.)}(\Omega)}=\|D f\|_{p(.)}$. The space $W_{0}^{1, p(.)}(\Omega)$ is separable and reflexive provided that with $1<p^{-} \leq p^{+}=\sup _{x \in \bar{\Omega}} p(x)<\infty$. 
The smooth functions are in general not dense in $W_{0}^{1, p(.)}(\Omega)$, but if the exponent variable $p()>$.1 satisfies the log-Hölder continuity condition (1.6), then the smooth functions are dense in $W_{0}^{1, p(.)}(\Omega)$.

For $u \in W_{0}^{1, p(.)}(\Omega)$ with $p \in C(\bar{\Omega},[1,+\infty))$, the Poincaré inequality holds (see [10])

$$
\|u\|_{p(.)} \leq C\|D u\|_{p(.)},
$$

for some constant $C$ which depends on $\Omega$ and the function $p$.

Lemma 2.1 ([8]). Let $\Omega \subset \mathbb{R}^{N}$ be an open bounded set, with Lipschitz boundary, and let $p: \bar{\Omega} \rightarrow(1, N)$ satisfy the log-Hölder continuity condition (1.6). Then we have the following continuous embedding:

$$
W^{1, p(.)}(\Omega) \hookrightarrow L^{p^{*}(.)}(\Omega)
$$

where $p^{*}()=.\frac{N p(.)}{N-p(.)}$.

The following Lemma will be used later.

Lemma 2.2 ([7]). If $\left(u_{n}\right), u \in L^{p(.)}(\Omega)$, then the following relations hold:

- $\|u\|_{p(.)}<1(>1 ;=1) \Leftrightarrow \rho_{p(.)}(u)<1(>1 ;=1) ;$

- $\min \left(\rho_{p(.)}(u)_{p^{+}}^{\frac{1}{2}} ; \rho_{p(.)}(u)_{p^{-}}^{\frac{1}{p^{-}}}\right)<\|u\|_{p(.)}<\max \left(\rho_{p(.)}(u)_{p^{+}}^{\frac{1}{2}} \rho_{p(.)}(u)_{p^{-}}^{\frac{1}{p^{-}}}\right)$

- $\|u\|_{p(.)} \leq \rho_{p(.)}(u)+1$;

- $\left\|u_{n}-u\right\|_{p(.)} \rightarrow 0 \Leftrightarrow \rho_{p(.)}\left(u_{n}-u\right) \rightarrow 0$

since $p^{+}<\infty$. 
Remark 2.1. Note that the following inequality:

$$
\int_{\Omega}|f|^{p(x)} d x \leq C \int_{\Omega}|D f|^{p(x)} d x
$$

in general does not hold (see [9]). But by Lemma 2.2 and (2.1), we have

$$
\int_{\Omega}|f|^{p(x)} d x \leq C \max \left\{\|D f\|_{L^{p(x)}(\Omega)}^{p^{+}} ;\|D f\|_{L^{p(x)}(\Omega)}^{p^{-}}\right\} .
$$

Throughout this paper, we denote by $C$ or $C_{i}, i=1,2, \ldots$ some generic positive constants independent of $n$.

\section{Statement of Results}

Definition 3.1. A function $u$ is a weak solution of problem (P) if

$$
u \in W_{0}^{1,1}(\Omega), \quad \widehat{a}(x, u, D u) \in\left(L^{1}(\Omega)\right)^{N},
$$

and

$$
\int_{\Omega} \widehat{a}(x, u, D u) D \varphi d x=\int_{\Omega} \varphi d \mu, \quad \forall \varphi \in C_{0}^{\infty}(\Omega) .
$$

Our main results are the following:

Theorem 3.1. Let $\mu \in M(\Omega)$ and assume that $p($.$) is restricted as in$ (1.4). Let $\hat{a}$ be a Carathéodory function satisfying (1.1)-(1.3). Then the problem $(\mathrm{P})$ has at least one weak solution $u \in W_{0}^{1, q(.)}(\Omega)$, where $q($.$) is a$ continuous function on $\bar{\Omega}$ satisfying

$$
1 \leq q(x)<\frac{N(p(x)-1)}{N-1} \text {, for all } x \in \bar{\Omega} .
$$

Now, we will improve the regularity of $\mu$ to obtain more summability of the gradients of solutions of $(\mathrm{P})$. 
Theorem 3.2. Let $\mu \in L^{m}(\Omega)$ and assume that $p($.$) and m$ are restricted as in (1.7)-(1.8). Let $\widehat{a}$ satisfying (1.1)-(1.3). Then the problem (P) has at least one weak solution $u \in W_{0}^{1, q(.)}(\Omega)$, where $q($.$) is a$ continuous function on $\bar{\Omega}$ satisfying

$$
1 \leq q(x)<\frac{N m(p(x)-1)}{N-m}, \text { for all } x \in \bar{\Omega} .
$$

Proof of Theorem 3.1. The proof needs three steps.

Step 1: Approximation.

Let $\left(\mu_{n}\right)$ be a sequence of $C_{0}^{\infty}(\Omega)$ which converges to $\mu \in M(\Omega)$ in $\mathcal{D}^{\prime}(\Omega)$ and verifies the inequality

$$
\left\|\mu_{n}\right\|_{L^{1}(\Omega)} \leq\|\mu\|_{M(\Omega)}, \quad \forall n \geq 1
$$

For $u \in W_{0}^{1, p(.)}(\Omega)$, we put

$$
A u=-\operatorname{div}(\widehat{a}(x, u, D u)) .
$$

The operator $A$ maps $W_{0}^{1, p(.)}(\Omega)$ into $\left(W_{0}^{1, p(.)}(\Omega)\right)^{\prime}$, thanks (1.3) $A$ is monotone. The growth condition (1.2) implies that $A$ is hemicontinuous, i.e., for all $u, v, w \in W_{0}^{1, p(.)}(\Omega)$, the mapping $\mathbb{R} \ni \lambda \mapsto\langle A(u+\lambda v), w\rangle$ is continuous. By (1.1) and Lemma 2.2, we can write

$$
\begin{aligned}
\frac{\langle A u, u\rangle}{\|u\|_{W_{0}^{1, p(.)}(\Omega)}} \geq & \alpha \frac{\rho_{p(.)}(D u)}{\|u\|_{W_{0}^{1, p(.)}(\Omega)}} \\
& \geq \alpha \frac{\min \left\{\|u\|_{W_{0}^{p^{+}}, p(.)(\Omega)},\|u\|_{W_{0}^{1, p(.)}(\Omega)}^{p^{-}}\right\}}{\|u\|_{W_{0}^{1, p(.)}(\Omega)}},
\end{aligned}
$$

this prove that $A$ is coercive. The operator $A$ is bounded. In fact, if $u \in W_{0}^{1, p(.)}(\Omega)$, we get 


$$
\begin{aligned}
\|A u\|_{\left(W_{0}^{1, p(.)}(\Omega)\right)^{\prime}} & \leq \sup _{\varphi \in W_{0}^{1, p(.)}(\Omega),\|\varphi\|_{W_{0}^{1, p(.)}(\Omega)} \leq 1} \int_{\Omega} \beta\left(h+|u|^{p(x)-1}+|D u|^{p(x)-1}\right)|D \varphi| d x \\
& \leq C_{1}\left\|\left(h+|u|^{p(x)-1}+|D u|^{p(x)-1}\right)\right\|_{\frac{p(.)}{p(.)-1}}
\end{aligned}
$$

using the fact that $\|u\|_{p(.)} \leq \rho_{p(.)}(u)+1$, we have

$$
\|A u\|\left(W_{0}^{1, p(.)}(\Omega)\right)^{\prime} \leq C_{2}+C_{3} \int_{\Omega}\left(h^{p^{\prime}(x)}+|u|^{p(x)}+|D u|^{p(x)}\right) .
$$

Thus, we get the desired result. A result given in [12] (or see [11]) shows that $A$ is surjective, and hence there exists a sequence $\left(u_{n}\right)_{n \in \mathbb{N}} \subset W_{0}^{1, p(.)}(\Omega)$ satisfying

$$
\int_{\Omega} \hat{a}\left(x, u_{n}, D u_{n}\right) D \varphi d x=\int_{\Omega} \mu_{n} \varphi d x, \quad \forall \varphi \in W_{0}^{1, p(.)}(\Omega) .
$$

Step 2: Uniform estimates.

Lemma 3.1. There exists a constant $C_{4}$ such that

$$
\int_{\Omega}\left|D T_{k}\left(u_{n}\right)\right|^{p(x)} d x \leq k C_{4}, \quad \forall k>0
$$

where $T_{k}$ is the function defined by

$$
T_{k}(v)= \begin{cases}k, & \text { if } v>k, \\ v, & \text { if }|v| \leq k, \\ -k, & \text { if } v<-k .\end{cases}
$$

Proof. Take $T_{k}\left(u_{n}\right)$ as a test function in (3.4). We find

$$
\begin{aligned}
\int_{\Omega} \widehat{a}\left(x, u_{n}, D u_{n}\right) D\left(T_{k}\left(u_{n}\right)\right) d x & =\int_{\Omega} \mu_{n} \cdot T_{k}\left(u_{n}\right) d x \\
& \leq k\|\mu\|_{M(\Omega)}
\end{aligned}
$$


Using (1.1) and the fact that $T_{k}^{\prime}=T_{k}^{\prime p(x)}$ for all $k>0$, we obtain

$$
\alpha \int_{\Omega}\left|D\left(u_{n}\right)\right|^{p(x)} \cdot T_{k}^{\prime}\left(u_{n}\right) d x \leq k\|u\|_{M(\Omega)},
$$

so that

$$
\int_{\Omega}\left|D T_{k}\left(u_{n}\right)\right|^{p(x)} d x \leq k C_{4}, \quad \forall k>0 .
$$

Lemma 3.2. Let $p($.) be a continuous function on $\bar{\Omega}$ and $\delta>1$. Then there exists a constant $C(\delta)>0$ independent of $n$, such that

$$
\int_{\Omega} \frac{\left|D u_{n}\right|^{p(x)}}{\left(1+\left|u_{n}\right|\right)^{\delta}} d x \leq C(\delta) .
$$

Proof. For fixed $\delta>1$, we define the function $\psi_{\delta}$ for all $t \in \mathbb{R}$ by

$$
\psi_{\delta}(t)=\int_{0}^{t} \frac{d x}{(1+|x|)^{\delta}},
$$

then we have $\psi_{\delta}(0)=0,0<\psi_{\delta}^{\prime} \leq 1$ and $\left|\psi_{\delta}\right| \leq \frac{1}{\delta-1}$. Taking $\psi_{\delta}\left(u_{n}\right)$ as a test function in (3.4) and using the last fact, we find

$$
\begin{aligned}
\alpha \int_{\Omega} \frac{\left|D u_{n}\right|^{p(x)}}{\left(1+\left|u_{n}\right|\right)^{\delta}} d x & \leq \int_{\Omega}\left|\mu_{n}\right| \cdot\left|\psi_{\delta}\left(u_{n}\right)\right| d x \\
& \leq \frac{1}{\delta-1} \int_{\Omega}\left|\mu_{n}\right| d x \\
& \leq \frac{\|\mu\|_{M(\Omega)}}{\delta-1}
\end{aligned}
$$

So that we have the proof of Lemma 3.2. 
Lemma 3.3. Let $p($.$) as in (1.4). Then there exists a constant C_{5}$ such that

$$
\left\|u_{n}\right\|_{W_{0}^{1, q(\cdot)}(\Omega)} \leq C_{5}
$$

for all continuous functions $q($.$) on \bar{\Omega}$ satisfying (3.1).

Proof. In a first step, let $q^{+}$be a constant satisfying

$$
q^{+}<\frac{N\left(p^{-}-1\right)}{N-1}<p^{-}
$$

Let us apply Hölder inequality, and Lemma 3.2, we obtain

$$
\begin{aligned}
\int_{\Omega}\left|D u_{n}\right|^{q^{+}} d x & =\int_{\Omega} \frac{\left|D u_{n}\right|^{q^{+}}}{\left(1+\left|u_{n}\right|\right)^{\delta} \frac{q^{+}}{p^{-}}}\left(1+\left|u_{n}\right|\right)^{\delta} \frac{q^{+}}{p^{-}} d x \\
& \leq\left(\int_{\Omega} \frac{\left|D u_{n}\right|^{p^{-}}}{\left(1+\left|u_{n}\right|\right)^{\delta}} d x\right)^{\frac{q^{+}}{p^{-}}} \cdot\left(\int_{\Omega}\left(1+\left|u_{n}\right|\right)^{\delta \frac{q^{+}}{p^{-}-q^{+}}} d x\right)^{1-\frac{q^{+}}{p^{-}}} \\
& \leq C_{\delta} \cdot\left(\int_{\Omega}\left(1+\left|u_{n}\right|\right)^{\delta \frac{q^{+}}{p^{-}-q^{+}}} d x\right)^{1-\frac{q^{+}}{p^{-}}} .
\end{aligned}
$$

Notice that (3.6) imply $\frac{q^{+}}{p^{-}-q^{+}}<q^{+\star}:=\frac{N q^{+}}{N-q^{+}}$. Then there exists $\delta>1$ such that $\delta \frac{q^{+}}{p^{-}-q^{+}}<q^{+\star}$. Using Poincaré-Sobolev inequality

$$
\begin{aligned}
\int_{\Omega}\left|D u_{n}\right|^{q^{+}} d x & \leq C_{6}+C_{7}\left(\int_{\Omega}\left|u_{n}\right|^{q^{+\star}} d x\right)^{1-\frac{q^{+}}{p^{-}}} \\
& \leq C_{6}+C_{8}\left(\int_{\Omega}\left|D u_{n}\right|^{q^{+}} d x\right)^{\frac{q^{+\star}}{q^{+}}\left(1-\frac{q^{+}}{p^{-}}\right)}
\end{aligned}
$$




$$
=C_{6}+C_{8}\left(\int_{\Omega}\left|D u_{n}\right|^{q^{+}} d x\right)^{\theta}
$$

where $\theta=\frac{N}{N-q^{+}}\left(1-\frac{q^{+}}{p^{-}}\right)$, by (1.4) we have $\theta \in(0,1)$. We deduce that

$$
\int_{\Omega}\left|D u_{n}\right|^{q^{+}} d x \leq C_{9}
$$

Since $\left|D u_{n}\right|^{q(x)} \leq\left|D u_{n}\right|^{q^{+}}+1$, we get

$$
\int_{\Omega}\left|D u_{n}\right|^{q(x)} d x \leq C_{10}
$$

In particular, there exists a constant $C_{11}>0$ such that

$$
\left\|u_{n}\right\|_{L^{1}(\Omega)} \leq C_{11} .
$$

In a second step, we suppose that (3.1) hold and

$$
q^{+} \geq \frac{N\left(p^{-}-1\right)}{N-1}
$$

By the continuity of $p(\cdot)$ and $q(\cdot)$ on $\bar{\Omega}$, there exists a constant $\delta>0$ such that

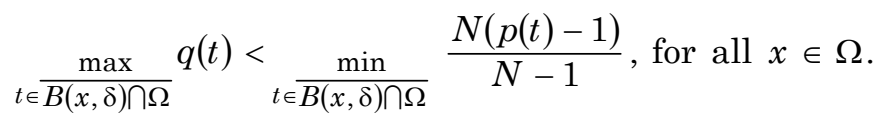

Note that $\bar{\Omega}$ is compact and therefore we can cover it with a finite number of balls $\left(B_{i}\right)_{i=1, \ldots, k}$. Moreover, there exists a constant $\nu>0$ such that

$$
\left|\Omega_{i}\right|=\operatorname{meas}\left(\Omega_{i}\right)>\nu, \Omega_{i}:=B_{i} \cap \Omega, \quad \text { for all } i=1, \ldots, k .
$$

We denote by $q_{i}^{+}$the local maximum of $q$ on $\overline{\Omega_{i}}$ (respectively, $p_{i}^{-}$the local minimum of $p$ on $\overline{\Omega_{i}}$ ), such that 


$$
q_{i}^{+}<\frac{N\left(p_{i}^{-}-1\right)}{N-1} \text {, for all } i=1, \ldots, k \text {. }
$$

By Poincaré-Wirtinger inequality, we have

$$
\left.\left\|u_{n}-\widetilde{u_{n}}\right\|_{L^{i_{i}}}^{q_{\left(\Omega_{i}\right)}} \leq C_{12}\left\|D u_{n}\right\|_{L^{i_{(}^{+}}} \Omega_{i}\right)
$$

with

$$
\widetilde{u_{n}}=\frac{1}{\left|\Omega_{i}\right|} \int_{\Omega_{i}} u_{n}(x) d x, \quad \text { and } \quad q_{i}^{+^{\star}}=\frac{N q_{i}^{+}}{N-q_{i}^{+}}
$$

From (3.8), (3.10), and (3.12), we deduce that

$$
\begin{aligned}
\left\|u_{n}\right\|_{L^{i_{i}^{+\star}}\left(\Omega_{i}\right)} \leq\left\|u_{n}-\widetilde{u_{n}}\right\|_{L^{q_{i}^{+\star}}\left(\Omega_{i}\right)}+\left\|\widetilde{u_{n}}\right\|_{L^{q_{i}^{+\star}}\left(\Omega_{i}\right)} \\
\leq C_{12}\left\|D u_{n}\right\|_{L^{q_{i}^{+}}\left(\Omega_{i}\right)}+C_{13} .
\end{aligned}
$$

Now, arguing locally as in (3.7), we obtain

$$
\int_{\Omega_{i}}\left|D u_{n}\right|^{q_{i}^{+}} d x \leq C_{14}+C_{15}\left(\int_{\Omega_{i}}\left|D u_{n}\right|^{q_{i}^{+}} d x\right)^{\theta_{i}}, \theta_{i}=\frac{N}{N-q_{i}^{+}}\left(1-\frac{q_{i}^{+}}{p_{i}^{-}}\right)<1,
$$

so that

$$
\int_{\Omega_{i}}\left|D u_{n}\right|^{q_{i}^{+}} \leq C_{16}, \quad \text { for all } i=1, \ldots, k
$$

Knowing that $q(x) \leq q_{i}^{+}$for all $x \in \Omega_{i}$, and all $i=1, \ldots, k$, we get

$$
\int_{\Omega_{i}}\left|D u_{n}\right|^{q(x)} d x \leq C_{16}+\left|\Omega_{i}\right|,
$$

so that

$$
\int_{\Omega}\left|D u_{n}\right|^{q(x)} d x \leq \sum_{i=1}^{k} \int_{\Omega_{i}}\left|D u_{n}\right|^{q(x)} d x=C_{17}
$$


where $C_{17}$ is a constant independent of $n$. This finishes the proof of the Lemma 3.3.

Step 3: Passage to the limit.

From Lemma 3.3 together with the continuous embedding $W_{0}^{1, q(.)}(\Omega) \hookrightarrow W_{0}^{1, q^{-}}(\Omega)$, we have a subsequence (still denoted $\left(u_{n}\right)$ ) such that

$$
\begin{gathered}
u_{n} \rightarrow u \quad \text { weakly in } W_{0}^{1, q^{-}}(\Omega), \\
u_{n} \rightarrow u \quad \text { strongly in } L^{q^{-}}(\Omega), \\
u_{n} \rightarrow u \quad \text { a.e. in } \Omega, \\
T_{k}\left(u_{n}\right) \rightarrow T_{k}(u) \quad \text { weakly in } W_{0}^{1, p(.)}(\Omega), \\
T_{k}\left(u_{n}\right) \rightarrow T_{k}(u) \quad \text { strongly in } \quad L^{p(.)}(\Omega) .
\end{gathered}
$$

To complete the proof, we need the following lemmas:

Lemma 3.4. We have

$$
D u_{n} \rightarrow D u \quad \text { a.e. in } \Omega .
$$

Proof. In order to prove this lemma it is sufficient to show that

$$
D u_{n} \rightarrow D u \quad \text { in measure. }
$$

Let $\lambda>0$, we set for some $\varepsilon>0$ and $k>0$, the following sets:

$$
\begin{aligned}
& E_{1}=\left\{x \in \Omega,\left|u_{n}\right|>k\right\} \cup\{x \in \Omega,|u|>k\} \cup\left\{x \in \Omega,\left|D u_{n}\right|>k\right\} \cup\{x \in \Omega,|D u|>k\}, \\
& E_{2}=\left\{x \in \Omega,\left|u_{n}-u\right|>\varepsilon\right\}, \\
& E_{3}=\left\{x \in \Omega,\left|u_{n}-u\right| \leq \varepsilon,\left|u_{n}\right| \leq k,|u| \leq k,\left|D u_{n}\right| \leq k,|D u| \leq k,\left|D u_{n}-D u\right| \geq \lambda\right\} .
\end{aligned}
$$

We remark that

$$
\left\{x \in \Omega,\left|D u_{n}-D u\right| \geq \lambda\right\} \subset E_{1} \cup E_{2} \cup E_{3} .
$$


From (3.15), for $\varepsilon>0$ fixed, we see that

$$
\operatorname{meas}\left(E_{2}\right) \rightarrow 0 \text { as } n \rightarrow+\infty
$$

We choose $k$ large enough in order to have meas $\left(E_{1}\right)$ arbitrarily small (this is possible by (3.14)). It remains to control meas $\left(E_{3}\right)$. Because the set $\left\{|s|,\left|\xi_{1}\right|,\left|\xi_{2}\right| \leq k,\left|\xi_{1}-\xi_{2}\right| \geq \lambda\right\}$ is a compact set and $(s, \xi) \mapsto \widehat{a}(x, s, \xi)$ is continuous for a.e. $x \in \Omega$, the quantity

$$
\left(\widehat{a}\left(x, s, \xi_{1}\right)-\widehat{a}\left(x, s, \xi_{2}\right)\right)\left(\xi_{1}-\xi_{2}\right)>0,
$$

reaches its minimum value on this compact set, and we will denote it by $w(x)$. Consequently, for any $\eta>0$, there exists $\eta^{\prime}>0$ such that

$$
\int_{E_{3}} w(x) d x<\eta^{\prime} \Rightarrow \operatorname{meas}\left(E_{3}\right) \leq \eta
$$

Hence, it is sufficient to show that for any given $\eta^{\prime}>0$ one can produce a small enough $\varepsilon>0$, such that

$$
\int_{E_{3}} w(x) d x<\eta^{\prime}
$$

By the definition of $w(x)$ and $E_{3}$, we can write

$$
\begin{aligned}
& \int_{E_{3}} w(x) d x \leq \int_{E_{3}}\left(\hat{a}\left(x, u_{n}, D u_{n}\right)-\widehat{a}\left(x, u_{n}, D u\right)\right)\left(D u_{n}-D u\right) d x \\
\leq & \int_{\left\{x:\left|u_{n}\right| \leq k,|u| \leq k\right\}}\left(\hat{a}\left(x, u_{n}, D u_{n}\right)-\hat{a}\left(x, u_{n}, D u^{k}\right)\right)\left(D u_{n}-D u^{k}\right) \chi_{\varepsilon}\left(\left|u_{n}-u^{k}\right|\right) d x \\
\leq & \int_{\Omega}\left(\widehat{a}\left(x, u_{n}, D u_{n}\right)-\hat{a}\left(x, u_{n}, D u^{k}\right)\right)\left(D u_{n}-D u^{k}\right) \chi_{\varepsilon}\left(\left|u_{n}-u^{k}\right|\right) d x \\
\leq & \int_{\Omega} \widehat{a}\left(u_{n}, D u_{n}\right) D T_{\varepsilon}\left(u_{n}-u^{k}\right)-\int_{\Omega} \widehat{a}\left(u_{n}, D u^{k}\right) D T_{\varepsilon}\left(u_{n}-u^{k}\right) \\
= & T_{n, \varepsilon}^{1}-T_{n, \varepsilon}^{2}
\end{aligned}
$$


where $\chi_{\varepsilon}$ denotes the characteristic function of $[0, \varepsilon] \subset[0,1]$. We begin with $T_{n, \varepsilon}^{2}$, that we write as

$$
\begin{aligned}
T_{n, \varepsilon}^{2} & =\int_{\Omega} \widehat{a}\left(x, u_{n}, D u^{k}\right)\left(D u_{n}-D u^{k}\right) \chi_{\varepsilon}\left(\left|u_{n}-u^{k}\right|\right) d x \\
& =\int_{\Omega} \widehat{a}\left(x, u_{n}^{k+1}, D u^{k}\right)\left(D u_{n}^{k+1}-D u^{k}\right) \chi_{\varepsilon}\left(\left|u_{n}^{k+1}-u^{k}\right|\right) d x
\end{aligned}
$$

Using Lemma 3.1, assumption (1.2), the fact that $p^{+}<\infty$, and Lebesgue's dominated convergence theorem, we obtain

$$
\begin{array}{r}
\widehat{a}\left(u_{n}^{k+1}, D u^{k}\right) \chi_{\varepsilon}\left(\left|u_{n}^{k+1}-u^{k}\right|\right) \rightarrow \widehat{a}\left(u^{k+1}, D u^{k}\right) \chi_{\varepsilon}\left(\left|u^{k+1}-u^{k}\right|\right) \\
\text { strongly in } L^{p^{\prime}(.)}(\Omega),
\end{array}
$$

and in view of (3.17), we get

$$
D u_{n}^{k+1}-D u^{k+1} \quad \text { weakly in } \quad L^{p(.)}(\Omega),
$$

so that

$$
\lim _{n} T_{n, \varepsilon}^{2}=\int_{\Omega} \widehat{a}\left(x, u^{k+1}, D u^{k}\right) D\left(u^{k+1}-u^{k}\right) \chi_{\varepsilon}\left(\left|u^{k+1}-u^{k}\right|\right) d x .
$$

Therefore, we conclude

$$
\lim _{\varepsilon \rightarrow 0} \lim _{n} T_{n, \varepsilon}^{2}=0
$$

To estimate $T_{n, \varepsilon}^{1}$, we choose $T_{\varepsilon}\left(u_{n}-u^{k}\right)$ as a test function in (3.4), we have

$$
\left|T_{n, \varepsilon}^{1}\right|=\left|\int_{\Omega} \mu_{n} T_{\varepsilon}\left(u_{n}-u^{k}\right) d x\right| \leq \varepsilon\|\mu\|_{M(\Omega)} \rightarrow 0 \text { as } \varepsilon \rightarrow 0
$$

uniformly in $n$. For $\varepsilon$ small enough, we have from (3.23), (3.24), and (3.25) that (3.22) holds, and by (3.21), also that meas $\left(E_{3}\right) \leq \eta$. Thus, we have the convergence of $\left(D u_{n}\right)$ to $(D u)$ in measure, which proves the Lemma. 
Lemma 3.5. We have

$$
\widehat{a}\left(x, u_{n}, D u_{n}\right) \rightarrow \widehat{a}(x, u, D u) \text { strongly in } L^{q(.)}(\Omega),
$$

for some continuous function $q():. \bar{\Omega} \rightarrow\left[1, \frac{N}{N-1}\right)$.

Proof. To prove (3.26), we apply Vitali's theorem. In one hand, by (3.16), (3.19) and the fact that $\widehat{a}$ is a Carathéodory function, we have

$$
a\left(x, u_{n}, D u_{n}\right) \rightarrow a(x, u, D u) \quad \text { a.e. in } \quad \Omega .
$$

On the other hand, for all continuous function $q():. \bar{\Omega} \rightarrow\left[1, \frac{N}{N-1}\right)$ and from (1.4), we can choose a continuous function $r($.$) on \bar{\Omega}$ such that: For all $x \in \bar{\Omega}$

$$
\left\{\begin{array}{l}
1 \leq q(x)<r(x)<\frac{N}{N-1}, \\
\frac{1}{p(x)-1}<r(x)<\frac{N}{N-1} .
\end{array}\right.
$$

Therefore, we have

$$
1<r(.)(p(.)-1)<\frac{N(p(.)-1)}{N-1} \text { and } r(.)<\frac{N}{N-1}<p^{\prime}(.) .
$$

Using (1.2), we get that the sequence $\left(\widehat{a}\left(x, u_{n}, D u_{n}\right)\right)_{n}$ is bounded in $L^{r(.)}(\Omega)$. Indeed, we have

$$
\begin{aligned}
& \int_{\Omega}\left|\widehat{a}\left(x, u_{n}, D u_{n}\right)\right|^{r(x)} d x \\
\leq & C_{18} 3^{r(x)-1}\left(\int_{\Omega}|h|^{r(x)} d x+\int_{\Omega}\left|u_{n}\right|^{r(x)(p(x)-1)} d x+\int_{\Omega}\left|D u_{n}\right|^{r(x)(p(x)-1)} d x\right) .
\end{aligned}
$$

Since $r()<.p^{\prime}($.$) , we get$

$$
\int_{\Omega}|h|^{r(x)} d x<\int_{\Omega}|h|^{p^{\prime}(x)} d x+|\Omega|<\infty .
$$


Using (3.27) together with Lemma 3.3, (2.2), and the Poincaré inequality, we find

$$
\int_{\Omega}\left|u_{n}\right|^{r(x)(p(x)-1)} d x<C_{19} \quad \text { and } \quad \int_{\Omega}\left|D u_{n}\right|^{r(x)(p(x)-1)} d x \leq C_{20},
$$

To prove the equi-integrability of $\widehat{a}\left(x, u_{n}, D u_{n}\right)$, using the Hölder's inequality and Lemma 2.2 together with the boundedness of $\widehat{a}\left(x, u_{n}, D u_{n}\right)$ in $L^{r(.)}(\Omega)$, we obtain

$$
\begin{aligned}
\int_{E}\left|\widehat{a}\left(x, u_{n}, D u_{n}\right)\right|^{q(x)} d x & \leq 2\left\|\left.\widehat{a}\left(x, u_{n}, D u_{n}\right)\right|^{q(x)}\right\| \frac{r(.)}{q(.)}\|1\|_{\left(\frac{r(.)}{q(.)}\right)^{\prime}} \\
& \leq C_{21} \max \left\{|E|^{\frac{1}{\omega^{+}}},|E|^{\frac{1}{\omega^{-}}}\right\}, \omega=\frac{r(.)}{r(.)-q(.)},
\end{aligned}
$$

which finish the proof of this Lemma.

Finally, for $\varphi \in C_{0}^{\infty}(\Omega)$, we have

$$
\int_{\Omega} \widehat{a}\left(x, u_{n}, D u_{n}\right) D \varphi d x=\int_{\Omega} \varphi \mu_{n}(x) .
$$

Using (3.26), we can pass to the limit for $n \rightarrow+\infty$ in the weak formulation (3.28) and we obtain that $u$ is a weak solution for (P).

Proof of Theorem 3.2. By proceeding as in Theorem 3.1, the proof needs three steps.

Step 1: Approximation.

By the density property, we can choose a sequence $\left(\mu_{n}\right) \subset C_{0}^{\infty}(\Omega)$

$$
\mu_{n} \rightarrow \mu \quad \text { strongly in } \quad L^{m}(\Omega) \text {, as } n \rightarrow \infty \text {, }
$$

such that

$$
\left\|\mu_{n}\right\|_{L^{m}(\Omega)} \leq\|\mu\|_{L^{m}(\Omega)}, \quad \forall n \geq 1
$$

Hence, by the same manner as above, the weak formulation (3.4) holds true for $\left(\mu_{n}\right)$ satisfy (3.29). 
Step 2: Uniform estimates.

We prove the following estimates:

Lemma 3.6. Let $p($.$) as in (1.7), and m$ as in (1.8). Then, for any constant $0<\delta<1$, there exists a constant $C_{\delta}$ independent of $n$ such that

$$
\int_{\Omega} \frac{\left|D u_{n}\right|^{p(x)}}{\left(1+\left|u_{n}\right|\right)^{\delta}} d x \leq C_{\delta}\left(1+\left(\int_{\Omega}\left(1+\left|u_{n}\right|\right)^{(1-\delta) \frac{m}{m-1}} d x\right)^{1-\frac{1}{m}}\right)
$$

Lemma 3.7. Let $p($.$) as in (1.7), m$ as in (1.8), and $\mu \in L^{m}(\Omega)$. Then there exists a constant $C_{22}$ such that

$$
\left\|u_{n}\right\|_{W_{0}^{1, q(.)}(\Omega)} \leq C_{22},
$$

for all continuous functions $q($.$) as in (3.2).$

Proof of Lemma 3.6. For any given $0<\delta<1$, we define the function $\psi_{\delta}: \mathbb{R} \rightarrow \mathbb{R}$ by

$$
\psi_{\delta}(t)=\int_{0}^{t} \frac{d x}{(1+|x|)^{\delta}}
$$

It's a clear that

$$
\psi_{\delta}(t)=\frac{1}{\delta-1}\left(1-\frac{1}{(1+|t|)^{\delta-1}}\right) \operatorname{sign}(t)
$$

where $\operatorname{sign}(t)$ denotes the sign of $t$. Note that $\psi_{\delta}$ is a continuous function satisfies $\psi_{\delta}(0)=0$, and $\left|\psi_{\delta}^{\prime}(\cdot)\right| \leq 1$. In the same way as in above, we take $\psi_{\delta}\left(u_{n}\right)$ as a test function in (3.4), we obtain

$$
\int_{\Omega} \hat{a}\left(x, u_{n}, D u_{n}\right) D \psi_{\delta}\left(u_{n}\right) d x=\int_{\Omega} \mu_{n} \psi_{\delta}\left(u_{n}\right) d x
$$

By (1.1) and using Hölder's inequality, we obtain 


$$
\begin{aligned}
& \alpha \int_{\Omega} \frac{\left|D u_{n}\right|^{p(x)}}{\left(1+\left|u_{n}\right|\right)^{\delta}} d x \leq\left\|\mu_{n}\right\|_{L^{m}(\Omega)} \cdot\left\|\psi_{\delta}\left(u_{n}\right)\right\|_{L^{m^{\prime}}(\Omega)} \\
& \leq C_{23}\left(\int_{\Omega}\left|\psi_{\delta}\left(u_{n}\right)\right| \frac{m}{m-1} d x\right)^{1-\frac{1}{m}} \\
& \left|\psi_{\delta}\left(u_{n}\right)\right|^{\frac{m}{m-1}} \leq C_{24}\left(1+\left(1+\left|u_{n}\right|\right)^{(1-\delta) \frac{m}{m-1}}\right) .
\end{aligned}
$$

So,

$$
\left(\int_{\Omega}\left|\psi_{\delta}\left(u_{n}\right)\right|^{\frac{m}{m-1}} d x\right)^{1-\frac{1}{m}} \leq C_{25}\left(1+\left(\int_{\Omega}\left(1+\left|u_{n}\right|\right)^{(1-\delta) \frac{m}{m-1}} d x\right)^{1-\frac{1}{m}}\right) .
$$

Hence,

$$
\int_{\Omega} \frac{\left|D u_{n}\right|^{p(x)}}{\left(1+\left|u_{n}\right|\right)^{\delta}} d x \leq C_{\delta}\left(1+\left(\int_{\Omega}\left(1+\left|u_{n}\right|\right)^{(1-\delta) \frac{m}{m-1}} d x\right)^{1-\frac{1}{m}}\right) .
$$

Proof of Lemma 3.7. Firstly, note that since $m>1$ and $p($.$) is$ defined as in (1.7), we get

$$
1<\frac{N m(p(x)-1)}{N-m}, \quad \text { for all } x \in \bar{\Omega}
$$

Now, consider the following cases:

Case (a): Let $q^{+}$be a constant satisfying

$$
q^{+}<\frac{N m\left(p^{-}-1\right)}{N-m}
$$

Note that the assumption (1.8) implies that 


$$
\frac{N m\left(p^{-}-1\right)}{N-m}<p^{-}
$$

Using Hölder's inequality with (3.30), we obtain

$$
\begin{aligned}
& \int_{\Omega}\left|D u_{n}\right|^{q^{+}} d x=\int_{\Omega} \frac{\left|D u_{n}\right|^{q^{+}}}{\left(1+\left|u_{n}\right|\right)^{\delta} \frac{q^{+}}{p^{-}}}\left(1+\left|u_{n}\right|\right)^{\delta \frac{q^{+}}{p^{-}}} d x \\
& \quad \leq\left(\int_{\Omega} \frac{\left|D u_{n}\right|^{p^{-}}}{\left(1+\left|u_{n}\right|\right)^{\delta}} d x\right)^{\frac{q^{+}}{p^{-}}} \cdot\left(\int_{\Omega}\left(1+\left|u_{n}\right|\right)^{\delta} \frac{q^{+}}{p^{-}-q^{+}} d x\right)^{1-\frac{q^{+}}{p^{-}}} \\
& \quad \leq C_{\delta}\left(1+\left(\int_{\Omega}\left(1+\left|u_{n}\right|\right)^{(1-\delta) \frac{m}{m-1}} d x\right)^{1-\frac{1}{m}}\right)^{\frac{q^{+}}{p^{-}}} \cdot\left(\int_{\Omega}\left(1+\left|u_{n}\right|\right)^{\delta \frac{q^{+}}{p^{-}-q^{+}}} d x\right)^{1-\frac{q^{+}}{p^{-}}} \\
& \leq C_{26}\left(1+\left(\int_{\Omega}\left(1+\left|u_{n}\right|\right)^{(1-\delta) \frac{m}{m-1}} d x\right)^{\left(1-\frac{1}{m}\right) \frac{q^{+}}{p^{-}}}\right) \cdot\left(1+\left(\int_{\Omega}\left(1+\left|u_{n}\right|\right)^{\delta \frac{q^{+}}{p^{-}-q^{+}}} d x\right)\right)^{1-\frac{q^{+}}{p^{-}}},
\end{aligned}
$$

so,

$$
\begin{aligned}
& \int_{\Omega}\left|D u_{n}\right|^{q^{+}} d x \leq \\
& C\left(1+\left(\int_{\Omega}\left(1+\left|u_{n}\right|\right)^{(1-\delta) \frac{m}{m-1}} d x\right)\right)^{\left(1-\frac{1}{m}\right) \frac{q^{+}}{p^{-}}} \cdot\left(1+\left(\int_{\Omega}\left(1+\left|u_{n}\right|\right)^{\delta} \frac{q^{+}}{p^{-}-q^{+}} d x\right)\right)^{1-\frac{q^{+}}{p^{-}}} .
\end{aligned}
$$

By (3.31) and (3.32), we get

$$
0<\frac{m\left(p^{-}-q^{+}\right)}{(m-1) q^{+}+m\left(p^{-}-q^{+}\right)}<1 .
$$


On the other hand, since the inequality $m<\frac{N p^{-}}{N p^{-}+p^{-}-N}$, is equivalent to $\frac{N m\left(p^{-}-1\right)}{N-m}<\frac{m N}{m N-N+m}$, by (3.31), we obtain $q^{+}<\frac{m N}{m N-N+m}$.

This inequality is equivalent to

$$
0<1-\left(\frac{N q^{+}}{N-q^{+}}\right)\left(\frac{m-1}{m}\right) .
$$

Also, (3.31), is equivalent to

$$
1-\left(\frac{N q^{+}}{N-q^{+}}\right)\left(\frac{m-1}{m}\right)<\frac{m\left(p^{-}-q^{+}\right)}{(m-1) q^{+}+m\left(p^{-}-q^{+}\right)} .
$$

Now, choose $\delta \in(0,1)$ such that

$$
\frac{\delta q^{+}}{p^{-}-q^{+}}<\frac{m(1-\delta)}{m-1}
$$

and

$$
\frac{m(1-\delta)}{m-1}<q^{+\star}:=\frac{N q^{+}}{N-q^{+}}
$$

Notice that (3.35) and (3.36) are, respectively, equivalent to

$$
\delta<\frac{m\left(p^{-}-q^{+}\right)}{(m-1) q^{+}+m\left(p^{-}-q^{+}\right)},
$$

and

$$
\delta>1-\left(\frac{N q^{+}}{N-q^{+}}\right)\left(\frac{m-1}{m}\right) .
$$

So, a such choice of $\delta \in(0,1)$ is possible since the inequalities (3.34) hold true. Therefore, by (3.33), we can write 


$$
\int_{\Omega}\left|D u_{n}\right|^{q^{+}} d x \leq C_{27}\left(1+\int_{\Omega}\left(1+\left|u_{n}\right|\right)^{(1-\delta) \frac{m}{m-1}} d x\right)^{1+\left(1-\frac{1}{m}\right) \frac{q^{+}}{p^{-}-\frac{q^{+}}{p^{-}}}}
$$

So that

$$
\int_{\Omega}\left|D u_{n}\right|^{q^{+}} d x \leq C_{28}\left(1+\int_{\Omega}\left|u_{n}\right|^{\left(\frac{1-\delta) m}{m-1}\right.} d x\right)^{1-\frac{q^{+}}{m p^{-}}} .
$$

Taking in consideration (3.36), the last inequality becomes

$$
\int_{\Omega}\left|D u_{n}\right|^{q^{+}} d x \leq C\left(1+\int_{\Omega}\left|u_{n}\right|^{q^{+\star}} d x\right)^{1-\frac{q^{+}}{m p^{-}}}
$$

Using Sobolev inequality with $q^{+\star}$, we obtain

$$
\begin{aligned}
\int_{\Omega}\left|D u_{n}\right|^{q^{+}} d x & \leq C_{29}\left(1+\int_{\Omega}\left|D u_{n}\right|^{q^{+}} d x\right)^{\left(\frac{N}{N-q^{+}}\right)\left(1-\frac{q^{+}}{m p^{-}}\right)} \\
& \leq C_{30}+C_{30}\left(\int_{\Omega}\left|D u_{n}\right|^{q^{+}} d x\right)^{\left(\frac{N}{N-q^{+}}\right)\left(1-\frac{q^{+}}{m p^{-}}\right)} .
\end{aligned}
$$

By the fact that

$$
m<\frac{N p^{-}}{N p^{-}-N+p}<\frac{N}{p^{-}}
$$

together with the assumption (3.31), this implies that

$$
q^{+}<m p^{-} \quad \text { and } \quad 0<\left(\frac{N}{N-q^{+}}\right)\left(1-\frac{q^{+}}{m p^{-}}\right)<1 .
$$

Hence, the estimate (3.40) imply that $\left(D u_{n}\right)$ is bounded in $L^{q^{+}}(\Omega)$. Since $\left|D u_{n}\right|^{q(x)} \leq\left|D u_{n}\right|^{q^{+}}+1$, we obtain that $\left(u_{n}\right)$ is bounded in $W_{0}^{1, q(.)}(\Omega)$. This completes the proof in Case (a). 
Case (b): Let $q$ be a continuous function satisfying (3.2) and

$$
q^{+} \geq \frac{N m\left(p^{-}-1\right)}{N-m}
$$

Now, similarly as in proof of Theorem 3.1, we have the following estimates as in (3.9), (3.10), and (3.11):

$$
\exists \eta>0, \max _{y \in \frac{\max }{B(x, \eta) \cap \Omega}} q(y)<\min _{y \in \overline{B(x, \eta) \cap \Omega}} \frac{N m(p(y)-1)}{N-m} \text {, for all } x \in \bar{\Omega} \text {. (3.42) }
$$

and

$$
\exists \rho>0, \quad\left|\Omega_{i}\right|>\rho, \quad \Omega_{i}:=B_{i} \cap \Omega, \quad \text { for all } i=1, \ldots, k,
$$

and

$$
q_{i}^{+}<\frac{N m\left(p_{i}^{-}-1\right)}{N-m}, \quad \text { for all } i=1, \ldots, k
$$

where $q_{i}^{+}=\max _{x \in \overline{\Omega_{i}}} q(x)$ and $p_{i}^{-}=\min _{x \in \overline{\Omega_{i}}} p(x)$. Using the same arguments as before locally, we obtain the similar estimate as in (3.39)

$$
\int_{\Omega_{i}}\left|D u_{n}\right|^{q_{i}^{+}} d x \leq C_{31}\left(1+\int_{\Omega_{i}}\left|u_{n}\right|^{q_{i}^{+^{\star}}} d x\right)^{1-\frac{q_{i}^{+}}{m p^{-}}} \text {, for all } i=1, \ldots, k .
$$

On the other hand, the Poincaré-Wirtinger inequality gives

$$
\left.\left\|u_{n}-\widetilde{u_{n}}\right\|_{L^{q_{i}}}{ }^{+*} \Omega_{i}\right) \leq C_{32}\left\|D u_{n}\right\|_{L^{i^{+}}\left(\Omega_{i}\right)},
$$

where

$$
\widetilde{u_{n}}=\frac{1}{\left|\Omega_{i}\right|} \int_{\Omega_{i}} u_{n} d x
$$

Moreover, note that the sequence $\left(u_{n}\right)_{n}$ is bounded in $L^{1}(\Omega)$. So, from (3.43), we have

$$
\left\|\widetilde{u_{n}}\right\|_{L^{1}(\Omega)} \leq C_{33}
$$


Therefore, by (3.46), we deduce that

$$
\begin{aligned}
\left\|u_{n}\right\|_{L^{q_{i}^{+\star}}\left(\Omega_{i}\right)} & \left.\leq\left\|u_{n}-\widetilde{u_{n}}\right\|_{L^{q_{i}}}{ }_{\left(\Omega_{i}\right)}+\left\|\widetilde{u_{n}}\right\|_{L^{q_{i}}}{ }^{\star_{(}} \Omega_{i}\right) \\
& \leq C_{32}\left\|D u_{n}\right\|_{L^{q_{i}^{+}}\left(\Omega_{i}\right)}+C_{34}, \quad \text { for all } i=1, \ldots, k .
\end{aligned}
$$

Thus, using (3.45), we obtain

$$
\int_{\Omega}\left|D u_{n}\right|^{q_{i}^{+}} d x \leq C_{35}+C_{35}\left(\int_{\Omega}\left|D u_{n}\right|^{q_{i}^{+}} d x\right)\left(\frac{N}{N-q_{i}^{+}}\right)\left(1-\frac{q_{i}^{+}}{m p_{i}^{-}}\right) .
$$

By (3.44) and arguing locally as in (3.41), we deduce

$$
0<\left(\frac{N}{N-q_{i}^{+}}\right)\left(1-\frac{q_{i}^{+}}{m p_{i}^{-}}\right)<1
$$

so that

$$
\int_{\Omega_{i}}\left|D u_{n}\right|^{q_{i}^{+}} \leq C_{36}, \quad \text { for all } i=1, \ldots, k
$$

Recall that

$$
q(x) \leq q_{i}^{+} \text {for all } x \in \Omega_{i}, \text { and all } i=1, \ldots, k .
$$

So, we get

$$
\int_{\Omega_{i}}\left|D u_{n}\right|^{q(x)} d x \leq \int_{\Omega_{i}}\left|D u_{n}\right|^{q_{i}^{+}} d x+\left|\Omega_{i}\right| \leq C_{37} .
$$

Since $\Omega \subset \bigcup_{i=1}^{i=k} \Omega_{i}$ for all $i=1, \ldots, k$, we deduce that

$$
\int_{\Omega}\left|D u_{n}\right|^{q(x)} d x \leq \sum_{i=1}^{k} \int_{\Omega_{i}}\left|D u_{n}\right|^{q(x)} d x=C_{38}
$$

This finishes the proof of the Case (b). 
To end the proof of Theorem 3.2, we need the following lemmas which are similar to (3.16), Lemmas 3.4 and 3.5. So, we omit their proofs.

Lemma 3.8.

$$
u_{n} \rightarrow u \quad \text { and } \quad D u_{n} \rightarrow D u \quad \text { a.e. in } \Omega \text {. }
$$

\section{Lemma 3.9.}

$$
\widehat{a}\left(x, u_{n}, D u_{n}\right) \rightarrow \widehat{a}(x, u, D u) \quad \text { strongly in } \quad L^{q(.)}(\Omega),
$$

for some continuous function $q: \bar{\Omega} \rightarrow\left[1, \frac{N m}{N-m}\right)$, where $m$ is as defined in (1.8).

Finally, we pass to the limit for $n \rightarrow+\infty$ in the weak formulation and we obtain that $u$ is a weak solution for $(\mathrm{P})$.

Remark 3.1. Remark that in the constant case and $\mu \in L^{m}(\Omega)$, we choose in (3.33),

$$
\delta=\frac{p N-m p-m N p+m N}{N-m p} \in(0,1)
$$

to obtain

$$
q=\frac{m N(p-1)}{N-m} \Rightarrow(1-\delta) \frac{m}{m-1}=\frac{\delta q}{p-q}=\frac{N q}{N-q} .
$$

It is easy to check that, instead of the global estimate (3.40), we find

$$
\int_{\Omega}\left|D u_{n}\right|^{q} d x \leq C+C\left(\int_{\Omega}\left|D u_{n}\right|^{q} d x\right)^{\left(\frac{N}{N-q}\right)\left(1-\frac{q}{m p}\right)},
$$

where

$$
0<\left(\frac{N}{N-q}\right)\left(1-\frac{q}{m p}\right)<1
$$


Then (P) has at least one weak solution $u$, possesses the regularity $u \in W_{0}^{1, q}(\Omega)$ for all $q=\frac{N m(p-1)}{N-m}$. For the nonconstant case, it remains an open problem to show that

$$
u \in W_{0}^{1, q(.)}(\Omega) \quad \text { with } \quad q(.)=\frac{N m(p(.)-1)}{N-m} .
$$

Remark 3.2. Note that the result given in Lemma 3.3 (resp., in Lemma 3.7) also holds for any measurable function $q: \bar{\Omega} \rightarrow \mathbb{R}$ such that

$$
\operatorname{ess} \inf _{\bar{\Omega}}\left(\frac{N(p(x)-1)}{N-1}-q(x)\right)>0\left(\text { resp., ess } \inf _{\Omega}\left(\frac{N m(p(x)-1)}{N-m}-q(x)\right)\right)>0
$$

Indeed, in both cases there exists a continuous function $s: \bar{\Omega} \rightarrow \mathbb{R}$ such that for almost every $x \in \bar{\Omega}$ :

$$
q(x) \leq s(x)<\frac{N(p(x)-1)}{N-1}\left(\text { resp., } q(x) \leq s(x)<\frac{N m(p(x)-1)}{N-m}\right) .
$$

From Lemma 3.3 (resp., Lemma 3.7), we deduce, in both cases, that $\left(u_{n}\right)$ is bounded in $W_{0}^{1, s(.)}(\Omega)$. Finally, by the continuous embedding $W_{0}^{1, s(.)}(\Omega) \hookrightarrow W_{0}^{1, q(.)}(\Omega)$ we have the desert result.

Remark 3.3. Under the assumption $\mu \in L^{m}(\Omega)$ in Theorem 3.2, we can deduce that $\mu$ is never in the dual space $\left(W_{0}^{1, p(.)}(\Omega)\right)^{\prime}$, so that the result of this paper deals with irregular data. If $m$ tends to be 1 , then $q()=.\frac{N m(p(.)-1)}{N-m}$ tends to be $\frac{N(p(.)-1)}{N-1}$. 


\section{References}

[1] E. Azroul, A. Barbara, M. B. Benboubker and S. Ouaro, Renormalized solutions for a $p(x)$-Laplacian equation with Neumann nonhomogeneous boundary conditions and

$L^{1}$-data, Annals of Univ. of Craiova, Mathematics and Computer Science Series 40 (2013), 9-22.

[2] M. Bendahmane and P. Wittbold, Renormalized solutions for nonlinear elliptic equations with variable exponents and $L^{1}$-data, Nonlinear Analysis TMA 70(2) (2009), 567-583.

[3] K. Bonzi Bernard, I. Nyanquini and S. Ouaro, Existence and uniqueness of weak and entropy solutions for homogeneous Neumann boundrary-value problems involving variable exponents, Electronic Journal of Differential Equations 12 (2012), 1-19.

[4] L. Boccardo and T. Gallouët, Nonlinear elliptic equations with right hand side measures, Comm. Partial Differential Equations 17 (1992), 641-655.

[5] L. Boccardo, T. Gallouët and L. Orsina, Existence and uniqueness of entropy solutions for nonlinear elliptic equations with measure data, Ann. Inst. H. Poincaré Anal. Non Linéaire 13(5) (1996), 539-551.

[6] Y. Chen, S. Levine and M. Rao, Variable exponent, linear growth functionals in image restoration, SIAM J. Appl. Math. 66 (2006), 1383-1406.

[7] L. Diening, P. Harjulehto, P. Hästö and M. Ruzicka, Lebesgue and Sobolev Spaces with Variable Exponents, Vol. 2017 of Lecture Notes in Mathematics, Springer, 2011.

[8] D. Edmunds and J. Rakosnik, Sobolev embeddings with variable exponent, Studia Math. 143 (2000), 267-293.

[9] X. L. Fan and D. Zhao, On the spaces $L^{p(x)}(U)$, and $W^{m, p(x)}(U)$, J. Math. Anal. Appl. 263 (2001), 424-446.

[10] P. Harjulehto, P. Hästö, M. Koskenoja and S. Varonen, The Dirichlet energy integral and variable exponent sobolev spaces with zero boundary values, Potential Analysis 25(3) (2006), 205-222.

[11] B. Lv, F. Li and W. Zou, Existence of weak solutions for some nonlinear elliptic equations with variable exponents, Complex Variables and Elliptic Equations 58 (2013), 1431-1447.

[12] J.-L. Lions, Quelques Méthodes de Résolution des Problémes aux Limites non Linéaires, Dunod, 1969.

[13] M. Mihailescu and V. Radulescu, A multiplicity result for a nonlinear degenrate problem arising in the theory of eletro-rheological fluids, Proc. R. Soc. A. 462 (2006), 2625-2641. 
[14] K. Rajagopal and M. Ruzicka, Mathematical modelling of electro-rheological fluids, Contin. Mech. Thermodyn. 13 (2001), 59-78.

[15] M. Ruzicka, Electrorheological fluids: Modeling and Mathematical Theory, Springer, Berlin, Lecture Notes in Mathematics 1748, 2000.

[16] M. Sanchon and J. M. Urbano, Entropy solutions for the $p(x)$-Laplace equation, Trans. Amer. Math. Soc. 361 (2009), 6387-6405.

[17] C. Zhang and S. Zhou, Entropy and renormalized solutions for the $p(x)$-Laplacian equation with measure data, Bull. Aust. Math. Soc. 82 (2010), 459-479.

[18] C. Zhang, Entropy solutions for nonlinear elliptic equations with variable exponents, Electronic Journal of Differential Equations 92 (2014), 1-14. 